\title{
Retinal Microscotomas Revealed with Adaptive-Optics Microflashes
}

\author{
Walter Makous, ${ }^{1,2}$ Joseph Carroll, ${ }^{1,3}$ Jessica I. Wolfing, ${ }^{1,4}$ Julianna Lin, ${ }^{1}$ Nathan Christie, ${ }^{5}$ \\ and David R. Williams ${ }^{1,2,4}$
}

Purpose. To develop a sensitive psychophysical test for detecting visual defects such as microscotomas.

Methods. Frequency-of-seeing curves were measured with $0.75^{\prime}$ and $7.5^{\prime}$ spots. On each trial, from 0 to 4 stimuli were randomly presented at any of eight equally spaced loci $0.5^{\circ}$ from fixation. By correcting the aberrations of the eye, adaptive optics produced retinal images of the $0.75^{\prime}$ spot that were $3.0 \mu \mathrm{m}$ wide at half height, small enough to be almost entirely confined within the typical cone diameter at this eccentricity. Data were collected from a patient with deuteranopia (AOS1) whose retina, imaged with adaptive optics, suggested that $\approx 30 \%$ of his cones were missing or abnormal. Patients with protanomalous trichromacy ( 1 subject), deuteranopia ( 1 subject), and trichromacy ( 5 subjects) served as controls (all had normal cone density and complete cone mosaics). Psychophysical results were modeled by a Monte Carlo simulation incorporating measured properties of the cone mosaic.

Results. Frequency-of-seeing curves for AOS1 obtained with $0.75^{\prime}$ spots showed lower asymptote, slope, and sensitivity than for controls. The $7.5^{\prime}$ results showed that these differences were the result of the small spot size, which on some trials was confined mostly to the locus of the putatively missing cones. A two-parameter model satisfactorily described the data and was highly sensitive to the proportion of missing cones simulated.

Conclusions. Adaptive-optics microperimetry is a powerful psychophysical test for assessing the loss of neural elements, even in retinas that appear otherwise normal in standard clinical tests. This technique may prove useful in estimating the proportion of missing cones in different patients and in detecting other visual losses such as those associated with glaucoma. (Invest Ophthalmol Vis Sci. 2006;47:4160 - 4167) DOI:10.1167/iovs.05-1195

From the ${ }^{1}$ Center for Visual Science, the ${ }^{2}$ Department of Brain and Cognitive Sciences, and the ${ }^{4}$ Institute of Optics, University of Rochester, Rochester, New York; and the ${ }^{5}$ University of North Carolina School of Medicine, Chapel Hill, North Carolina.

${ }^{3}$ Present affiliation: Department of Ophthalmology, Medical College of Wisconsin, Milwaukee, Wisconsin.

Presented, in part, at the 2005 OSA Fall Vision Meeting in Tuscon, Arizona, October 21-23.

Supported by National Eye Institute Grants R01-EY04367, P30EY01319, and F32-EY014749; and in part by the National Science Foundation Science and by the Technology Center for Adaptive Optics, managed by the University of California at Santa Cruz under Cooperative Agreement AST-9876783.

Submitted for publication September 7, 2005; revised February 23 and April 3, 2006; accepted July 3, 2006.

Disclosure: W. Makous, None; J. Carroll, None; J.I. Wolfing None; J. Lin, None; N. Christie, None; D.R. Williams, P, C

The publication costs of this article were defrayed in part by page charge payment. This article must therefore be marked "advertisement" in accordance with 18 U.S.C. $\$ 1734$ solely to indicate this fact

Corresponding author: Walter Makous, Center for Visual Science, University of Rochester, Box 270268, Rochester, NY 14627-0270; walt@cvs.rochester.edu.
Tnsults to the visual system are notoriously difficult to detect. For example, in human glaucomatous eyes, before a deficit in visual performance can be detected by standard automated perimetry, $25 \%$ to $35 \%$ of the ganglion cells are dead, ${ }^{1}$ and many more may be nonfunctional or of reduced sensitivity. The redundancy of visual mechanisms explains part of the insensitivity of such tests ${ }^{2-6}$ because the sensitivity profiles of many neurons overlap along the stimulus dimensions to which they respond, so that when one neuron dies or otherwise fails to respond to a given stimulus, the stimulus can nevertheless be detected through the response of neighboring neurons. Although newer techniques, such as short-wavelength automated perimetry, ${ }^{7}$ frequency-doubling perimetry, ${ }^{8,9}$ high-pass resolution perimetry, ${ }^{10}$ and other techniques, ${ }^{2}$ diminish the redundancy in the neural mechanisms, the stimuli themselves are redundant enough to cause correlated responses even in neurons whose sensitivities do not overlap. For example, the smallest spots used in clinical perimetry subtend $0.11^{\circ}$. Even without optical spread, the image of such a spot covers some 150 cones at the center of the fovea of an average retina. ${ }^{11}$ This produces correlated responses not only among these 150 cones but also among the many other postreceptoral cells connected to them either directly or indirectly. One way to reduce the redundancy of the neural response is to reduce the component caused by the test stimulus itself. Normally, spread of the retinal image by the optics of the eye limits the amount by which one can reduce the stimulus size and hence the number of cones excited. Here we used adaptive optics to reduce the size of the retinal image close to the theoretical limit and thereby minimize the number of cones stimulated on any given presentation; we used these small test spots to detect missing cones in a retina in which larger spots used in clinical perimetry could not.

The person whose retina we tested (AOS1) represented a subclass of persons with dichromacy who carry genetic mutations that disrupt the function of one of the cone photopigments. ${ }^{12}$ Previous results with high-resolution retinal imaging $^{13}$ have shown that the retina of AOS1 contained lacunae, equal in size to one or more cones, that the authors hypothesized represented the loci at which cones expressing the mutant gene have been severely damaged or lost. A micrograph of his retina is shown in the upper part of Figure 1; for comparison, the retina of a healthy trichromat (AOS2) is shown below it. Note the relative sparseness of visible cones in the upper micrograph. Although the hypothesized missing cones occupy almost a third of the cone mosaic, ophthalmic examination revealed no abnormalities aside from the dichromacy. For example, visual acuity was 20/16 ( $-0.10 \log$ MAR), standard visual fields were normal, and funduscopic examination results were unremarkable. Test stimuli used here were small enough to fall largely within the lacunae observed photographically and thereby made it possible to detect the associated microscotomas psychophysically. Use of large spots, approximating the size of those in clinical use, failed to produce the signs we attributed to the missing cones. 


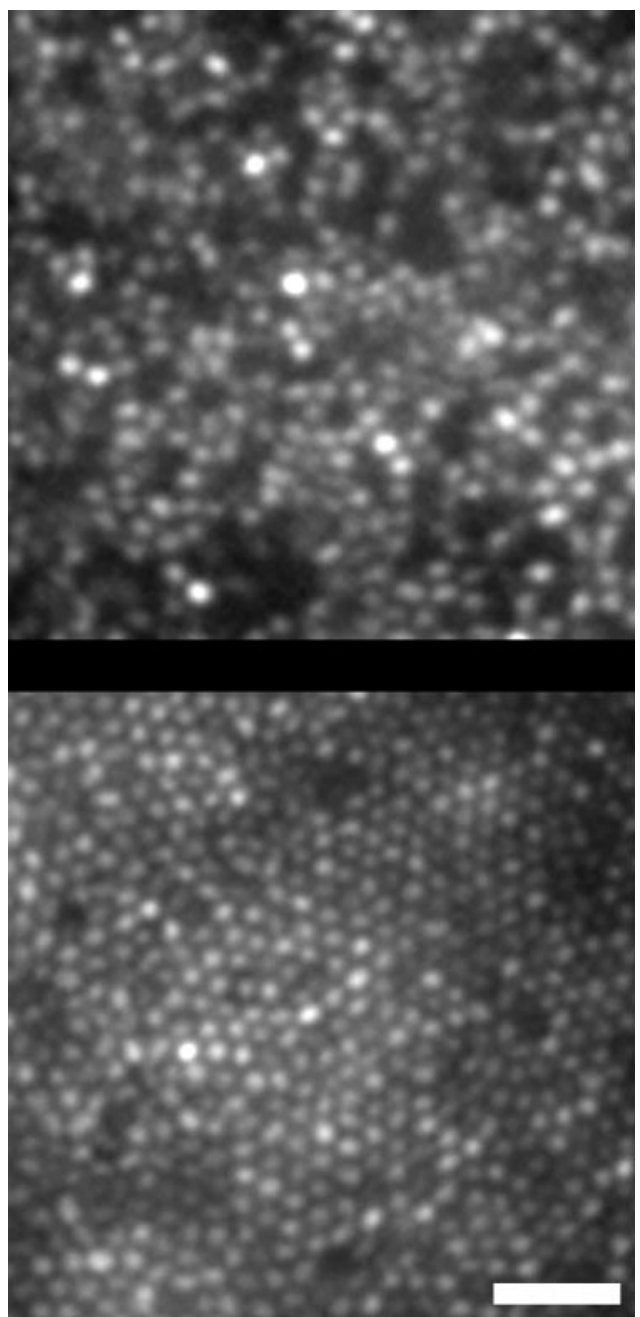

FIGURE 1. High-resolution retinal images obtained with an adaptive optics ophthalmoscope. Top: deuteranope, AOS1 (age 26); bottom: trichromat, AOS2 (age 29). Each image is a registered sum of six individual frames and was taken from the right eye at approximately $0.5^{\circ}$ from fixation. Scale bar, $20 \mu \mathrm{m}$.

\section{Methods}

\section{Subjects}

A mutation in the gene for middle-wavelength-sensitive (M) pigment opsin in AOS1 prevents it from functioning properly, ${ }^{13,14}$ and the resultant phenotype is deuteranopic. As shown in Figure 1, AOS1 has a patchy loss of normal cones throughout the photoreceptor mosaic. ${ }^{13}$ The apparent gaps in retinal mosaic, representing approximately $30 \%$ of the cone mosaic, are randomly distributed among the visible cones. ${ }^{13}$ The visual field of AOS1, as measured by a Humphrey (Allergan Humphrey, San Leandro, CA) 10-2 test with a size III spot, was in all respects completely normal. A second person with deuteranopia, another with protanomalous trichromacy, and five with trichromacyall seven with normal cone mosaics-served as controls. Classification of color vision was based on performance on anomaloscopic color matches and AO-HRR pseudoisochromatic plates. The control deuteranope and protanomalous trichromat were examined genetically and showed no mutations in the $\mathrm{M}$ or the long wavelength-sensitive (L) pigment that have been linked to a loss of photopigment function; rather, the $\mathrm{M}$ gene was deleted in the deuteranope and the $\mathrm{L}$ gene was replaced by a second $\mathrm{M}$ gene in the protanomalous trichromat (Neitz M, personal communication, 2005). Ophthalmic examination results on all subjects revealed no abnormalities. All subjects provided in- formed consent after the nature and possible consequences of the study were explained. All research followed the tenets of the Declaration of Helsinki, and study protocols were approved by the institutional research subjects review board of the University of Rochester.

\section{Stimulus Presentation}

One drop each of phenylephrine hydrochloride (2.5\%) and tropicamide (1\%) dilated the subjects' pupils and suspended accommodation in their right eyes. Their heads were stabilized by a bite bar containing their dental impression. Stimuli were $0.75^{\prime}$ or $7.5^{\prime}$ spots produced by a digital light-processing (DLP) video projector (MP1600; Compaq; Hewlett Packard, Mountain View, CA $)^{15}$ at any of eight equally spaced locations $0.5^{\circ}$ from a dim, $2^{\prime}$ fixation target (Fig. 2). The light passed through an interference filter with a $10-\mathrm{nm}$ pass band centered at 550 $\mathrm{nm}$, a wavelength to which $\mathrm{M}$ and $\mathrm{L}$ cones are equally sensitive. At maximum, the test spot illuminated the retina at $5.06 \mathrm{log}$ troland. The projector also provided a steady background field of $3.08 \mathrm{log}$ troland (its minimum output) covering a circular region slightly greater than $1^{\circ}$. Sensitivity to the $7.5^{\prime}$ spot was so much greater than that to the $0.75^{\prime}$ spot that a $1.11 \mathrm{log}$ filter was required in the projector beam to present spots dim enough to measure the low end of the frequencyof-seeing curve. This brought the maximum $\log$ troland value down to 3.95. Because the filter also reduced the background field, we added a steady, 550-nm background field that brought the total background field for the $7.5^{\prime}$ spots back up to $3.44 \log$ troland, comparable to that for the $0.75^{\prime}$ spots.

The shortest duration flash allowed by the proprietary software of the projector delivered a train of six pulses of equal intensity: each pulse was $3 \mathrm{~ms}$ long, and each was separated from one another by 5.5 ms of darkness. Each of the 3-ms pulses was in turn composed of micropulses that were turned on and off by the projector's software to vary the total amount of light contained by the six pulses over 256 steps. Troland values of the stimuli used for each subject are shown by the locations of the data points (see Fig. 4).

A spectro-colorimeter (PR-650; Photograph Research, Chatsworth, CA) was used to calibrate luminous flux in the test stimuli, and a radiometer (IL 1700; International Light, Newport, MA) was used to measure the power of the background, which was numerically converted to trolands. These measurements were made in the plane of the subject's pupil. A high-speed photodiode (PDA 155; Thorlabs, Newton, NJ) and oscilloscope were used to measure temporal properties of the DLP projector output.

Because the time available for testing AOS1 was limited, we increased the rate of data acquisition by simultaneously presenting 0,1 , 2,3 , or 4 stimuli with equal frequency as is sometimes done in clinical perimetry. ${ }^{16-18}$ Although one can further increase efficiency by presenting four stimuli on a higher proportion of trials, we chose not to so as to avoid the response biases that might result. The subject's task was to report, by a key-press, the number of stimuli presented. This increase in the number of stimuli and alternative responses only slightly delays the response $\mathrm{e}^{19,20}$ and has no noticeable effect on the reliability of detection $^{19,21-23}$ (our results also show no relationship between a
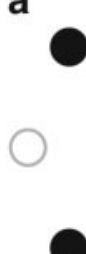

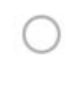

○
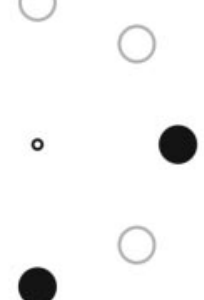

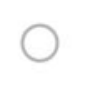

Figure 2. Test stimuli. See "Stimulus Presentation" for the details. Black disks: four simultaneously presented stimuli; open gray circles: other possible stimulus locations; center open circles: fixation disk. Stimuli are $7.5^{\prime}$ in diameter (a) and $0.75^{\prime}$ in diameter (b). 
detection and number of stimuli). The subject's response initiated the next trial.

The stimuli described were presented through an adaptive optical system that minimized optical aberrations. ${ }^{24,25}$ Measured point-spread functions with and without adaptive optics are shown in Figure 3. Although cone sizes varied over the areas tested and among subjects, as a rough estimate, without adaptive optics approximately $9 \%$ of the incident light in a $0.75^{\prime}$ test spot fell within the geometric boundaries of a single cone, whereas with adaptive optics approximately $55 \%$ did so that adaptive optics increased the amount of light in this area approximately sixfold. Because of the Gaussian shape of the cone entrance aperture, this optical correction increased the relative amount of light that actually entered the cone even more.

Preliminary observations established the range spanned by each observer's frequency-of-seeing curve, and from five to eight points on the curve, spaced at 0.1 intervals on a log troland scale, were tested to describe the curve. A block of trials consisted of 50 trials at each stimulus magnitude, in random order, and each observer had four such blocks. Measurement and correction of the aberrations of each sub-

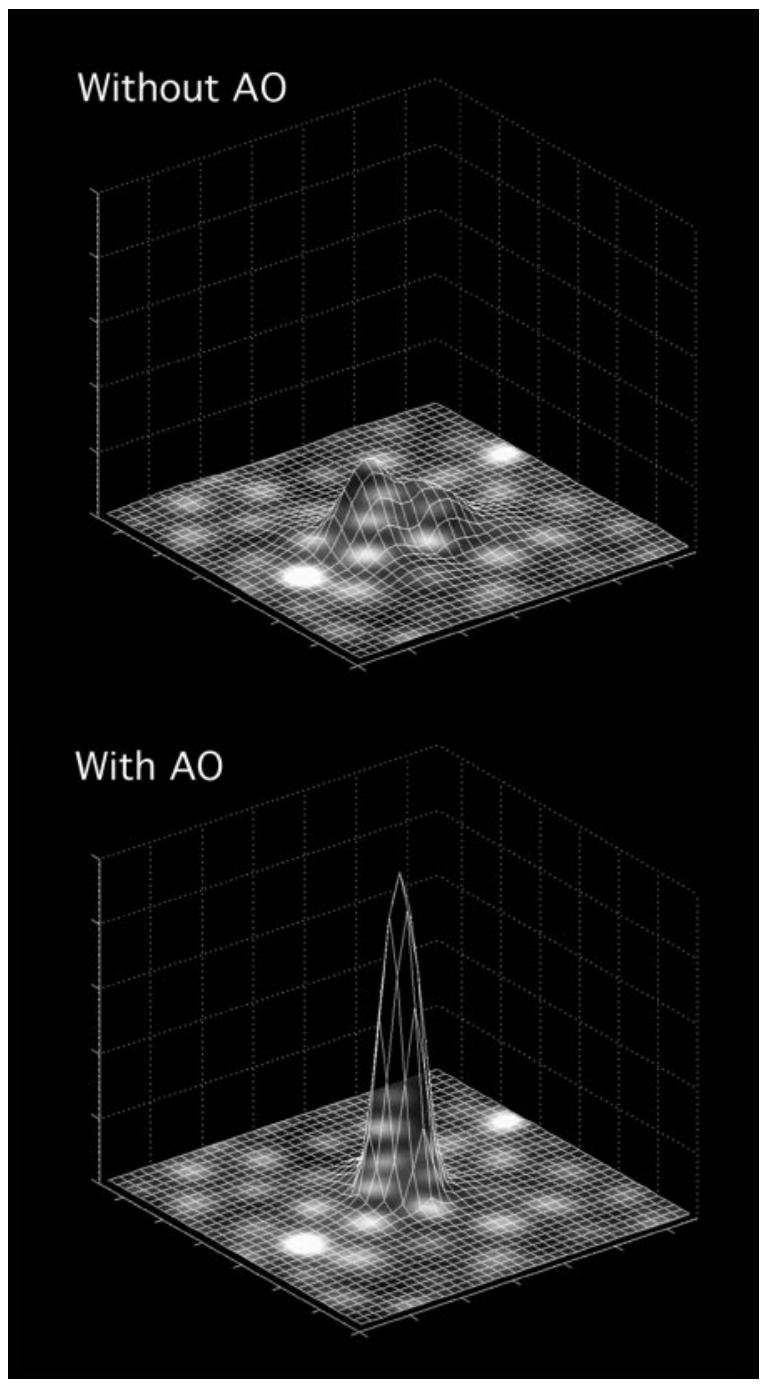

FiguRE 3. Retinal stimulus profiles for a $0.75^{\prime}$ spot at $0.5^{\circ}$ from fixation without (top) and with (bottom) correction of higher-order aberrations of the eye of AOS2 with adaptive optics. The profile without adaptive optics was optimized by varying defocus to maximize the Strehl ratio. The width of the spot corrected with adaptive optics, spread by diffraction and residual aberrations, is $3.0 \mu \mathrm{m}$ at half height. The white spots, taken from a small patch in the lower part of Figure 1 , represent cones. ject's eye was performed between each block of trials, instead of during the blocks, to prevent the superluminescent diode used for wavefront sensing from affecting the subject's sensitivity. Although the measurement took less than $800 \mathrm{~ms}$, more than 1 minute was required for the subject's eye to recover from exposure to the light from the diode.

\section{Model}

To determine whether the differences between the $0.75^{\prime}$ data of AOS1 and of the control subjects could be explained on the basis of the evident differences in their retinal mosaic, we simulated the experiment with a model (implemented in MATLAB; MathWorks, Natick, MA) that incorporates the retinal mosaic and optical point-spread functions of AOS1 and that of one of the trichromatic subjects (AOS2).

The model consisted of a Monte Carlo simulation of 10,000 stimuli of varying energy, presented to a given subject's retinal cone mosaic. For each stimulus presentation, the model used a set of detection rules to determine whether the stimulus was seen. The output of the model was a frequency-of-seeing curve.

According to the model, a given stimulus was detected if the visual excitation exceeded the response criterion for that flash. Visual excitation was calculated by summing the products of each point of the retinal image of the spot with the corresponding point of the sensitivity profile of the photoreceptor mosaic (i.e., by finding the inner product). The retinal image of the spot was found by convolving the geometric image of the stimulus with the subject's own point spread function, measured at an eccentricity of $0.5^{\circ}$, after the aberrations of that subject's eye had been optically compensated. ${ }^{24,25}$

The sensitivity profile of the photoreceptor mosaic was obtained by convolving the optical aperture of the cones with a set of points representing locations of the observed centers of the cones in a patch of retina approximately $0.75^{\circ}$ square that was imaged in vivo at $0.5^{\circ}$ eccentricity by an adaptive optics ophthalmoscope. ${ }^{26}$ Individual cones were assigned a spectral sensitivity based on the relative numbers of short-wavelength-sensitive (S), M, and $\mathrm{L}$ cones in the retina. Because these vary across healthy subjects, we used previous densitometry measurements with an adaptive optics ophthalmoscope ${ }^{13,24,27,28}$ to determine the appropriate ratio of S-, M-, and L-cone assignments to use for each subject. In addition, the optical aperture of each cone was represented by a two-dimensional Gaussian profile with a width at half height of 0.615 times the intercone distance, on the basis of interferometric estimates. ${ }^{29-32}$ We truncated the Gaussian at the physical boundary of the cone (meaning that light falling on the cone mosaic outside the cone's physical aperture would not be absorbed by photopigment within that particular cone), but doing so had a negligible effect on the results (data not shown).

Because of fixational eye movements, the absolute location of a stimulus on the retina varied from trial to trial. Therefore, we incorporated fixational instability in the model to determine the location of the retinal mosaic on which the stimulus fell on each trial. The position of the retinal mosaic with respect to the retinal image of the spot was sampled randomly from a probability density function representing the statistics of eye position measurements. The two-dimensional distribution of fixations of AOS2 and four other subjects were observed by using high-resolution imaging with adaptive optics to record the absolute position of the retina while fixating. ${ }^{24}$ The fixational patterns of AOS2 so obtained were used in the simulations of his psychophysical performance, whereas the mean of all five subjects was used in the simulation the performance of AOS1. Consistent with previous reports using a different methodology, in both cases the fixational patterns were elongated along the horizontal axis and had an SD of approximately $5^{\prime}$.

The model therefore yields on each trial an estimate of the relative amount of light absorbed by each cone in the vicinity of the test flash. Although previous models of the detection of small spots by the cone system $^{33-42}$ have assumed that each cone has a threshold, in the sense that stimulation of a cone below a fixed value makes no contribution 
FigURE 4. Frequency-of-seeing curves for the $0.75^{\prime}$ (left) and $7.5^{\prime}$ (right) test spots. Filled black circles: AOS1; filled gray squares and triangles: control deuteranope and protanomalous trichromat, respectively; open symbols: control trichromats. Curves: maximum likelihood fits of cumulative Gaussians; dashed lines: trichromats; solid gray: control deuteranope and protanomalous trichromat; solid black: AOS1. Horizontal dashed lines: estimated asymptotes for AOS1 and the mean of the estimated asymptotes for the controls.

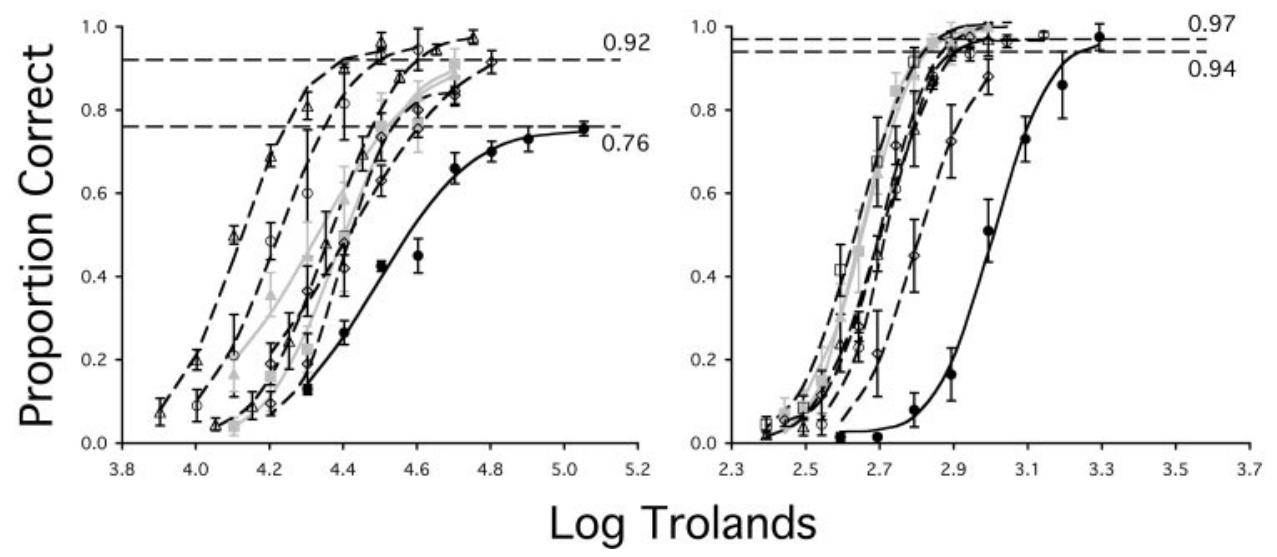

to detection by the subject, we found such a threshold unnecessary; excluding it simplifies the model while improving the fit slightly (though not reliably). We assumed instead that the total number of photopigment molecules activated (visual excitation) in all the cones sums linearly to determine whether the flash was seen: if the total number of activations exceeded a given value, the response criterion, we assumed that the subject detected the stimulus. (Nonlinear summation of cone excitation, such as root-mean-square summation, ${ }^{43-46}$ yields frequency-of-seeing curves with too great a slope.) To obtain frequency-of-seeing curves, we simply counted the proportion of trials on which the visual excitation exceeded the response criterion at each of the stimulus intensities.

The model thus has two free parameters that were separately adjusted to fit each subject's data: the observer's response criterion, just mentioned, and sensitivity, the proportion of quanta in the stimulus that ultimately activates a photopigment molecule. Sensitivity simply relates such visual excitation to the amount of light absorbed, and changing its value translates the frequency-of-seeing curve along the $\log$ intensity axis. The response criterion is the visual excitation required for the subject to respond to the stimulus; changing its value translates the frequency-of-seeing curve along the absolute intensity axis and varies the slope and shape of the frequency-of-seeing curve when plotted on semilog axes. Thus, in the model, two parameters serve where three were required for the curves in Figure 4.

In our simulations, variation in the locations of the spots relative to the cones produced by eye movements caused variation in visual excitation from trial to trial, depending on where the spots fell with respect to the cones and gaps between cones in the mosaic. Increasing the response criterion increased the number of trials in which too little light was absorbed to be detected. This affected both the height and the slope of the frequency-of-seeing curve. Hence, the topography of the individual's retinal mosaic determined the trial-by-trial variability, and the subject's response criterion determined the effect of that variability on the frequency-of-seeing curve.

The variability of light absorbed qualifies as noise, and so the model is based on the theory of signal detection. ${ }^{4-49}$ However, in the experiments using the $7.5^{\prime}$ spot, the variability of absorbed light resulting from variation in spot location was negligible. The stochastic noise associated with quantal absorptions was also small, approximately $0.3 \%$ of the mean (SD). Hence, the trial-by-trial variability of the response has to be attributed to some other source, such as noise intrinsic to the visual system. For AOS2 this inferred noise was 22 times smaller than that for the smaller spot, and for AOS1 it was 115 times smaller. The contribution of such visual noise to detection of the small spot was therefore small relative to that associated with stimulus location. Nevertheless, for the sake of consistency, we included this noise in the model for detection of the small spot, assuming that the amount of intrinsic noise involved in detecting the small spot was the same as that for the large spot. This was incorporated into the model by adding a random sample from a Gaussian distribution to the total visual excitation on each trial.
The model is of course somewhat simplified. For example, although the intercone distances in our model faithfully reflected those of the individual retina tested, we assumed a fixed cone size. Similarly, the stimuli actually fell at eight locations, but our model represented the cone mosaic at only one location: there can be small differences in cone density on different meridians, ${ }^{50,51}$ and the effects of any such asymmetries could be exaggerated by the tendency of observers to fixate off the anatomic center of the fovea. ${ }^{24}$

The model also omitted absorption of light by the retinal blood vessels. Approximately $90 \%$ of the retina is covered with a capillary bed that absorbs some $40 \%$ of incident light. ${ }^{52}$ Placing the stimuli $0.5^{\circ}$ from fixation, an area that in most persons is devoid of retinal vasculature, mitigates this problem. However the size of this avascular zone varies widely, and its radius in healthy persons can be as small as $0.3^{\circ} .^{53}$ Indeed, retinal imaging reveals that subject AOS6 in this experiment has extensive invasion of the central fovea by retinal vessels (data not shown). Therefore, one would expect the slope of his frequency-ofseeing curve to be shallower than those of the other subjects and also shallower than predicted by the model. That this subject does have the shallowest frequency-of-seeing curve is evident in Table 1 and Figure 4, and it is significantly shallower than that generated by the model for his retina (data not shown).

\section{Results}

\section{Psychophysical Data}

Figure 4 shows the results obtained with the small and large test spots, and Table 1 provides a summary of the relevant statistics. The curves in Figure 4 are maximum likelihood fits of a cumulative Gaussian curve to the data. The means, slopes at the means, and upper asymptotes of those curves are given in Table 1. The means are used as an estimate of each observer's psychophysical threshold for detecting the test stimulus and, for the control subjects, are comparable to those in the literature. ${ }^{54-56}$ False-positive rate, $f_{+}$, for a particular subject is specified by the equation:

$$
f_{+}=d_{\mathrm{r}} /\left(8 N_{b}\right)
$$

where $d_{\mathrm{r}}$ is the number of reported detections when no stimulus was presented, $N_{\mathrm{b}}$ is the number of trials on which no stimulus was presented (blank trials), and 8 is the number of possible locations for the stimulus on each trial. We assumed that the number of true detections, $d$, is:

$$
d=d_{\mathrm{r}}-f_{+}(8 N-d)
$$

where $N$ is the total number of trials. Here, the false-positive rate $\left(f_{+}\right)$is multiplied by the number of opportunities for a 
TABLE 1. Statistical Summary of Frequency-of-Seeing Data

\begin{tabular}{|c|c|c|c|c|c|c|c|c|}
\hline \multirow[b]{2}{*}{ Subject } & \multicolumn{4}{|c|}{$0.75^{\prime}$ Spot } & \multicolumn{4}{|c|}{ 7.5' Spot } \\
\hline & Mean & Slope & Asymptote & False+ & Mean & Slope & Asymptote & False+ \\
\hline AOS1 & $4.50^{*}$ & $3.28^{*}$ & $0.76 \dagger$ & 0.0023 & $3.01 \dagger$ & 7.81 & 0.95 & 0.0068 \\
\hline AOS2 & 4.12 & 6.53 & 0.93 & 0.0043 & 2.64 & 8.04 & 1.00 & 0.0026 \\
\hline AOS3 & 4.40 & 7.70 & 0.85 & 0.0000 & 2.80 & 7.55 & 0.93 & 0.0008 \\
\hline AOS 4 & 4.37 & 6.27 & 0.97 & 0.0029 & 2.71 & 7.42 & 0.99 & 0.0008 \\
\hline AOS5 & 4.22 & 5.87 & 0.95 & 0.0045 & 2.72 & 10.11 & 0.95 & 0.0058 \\
\hline AOS6 & 4.42 & 3.81 & 0.94 & 0.0083 & 2.71 & 9.03 & 0.97 & 0.0107 \\
\hline AOS7 & 4.39 & 5.91 & 0.91 & 0.0018 & 2.65 & 9.97 & 0.99 & 0.0009 \\
\hline AOS8 & 4.33 & 3.89 & 0.89 & 0.0111 & 2.67 & 8.96 & 0.96 & 0.0120 \\
\hline Mean & 4.32 & 5.71 & 0.92 & 0.0047 & 2.70 & 8.72 & 0.97 & 0.0048 \\
\hline $\mathrm{SE}$ & 0.041 & 0.533 & 0.015 & 0.0014 & 0.021 & 0.414 & 0.010 & 0.0018 \\
\hline
\end{tabular}

Mean and SE values are for the seven controls. False + indicates rate of false-positive responses. Units are given in log trolands.

* Statistically significant at $P<0.01$, compared with controls

† Statistically significant at $P<0.001$, compared with controls.

false positive, which is eight times the number of trials minus the number of true detections $(d)$. Dividing $d$ by the number of stimuli presented yields proportion correct, as plotted in Figures 4 to 6 .

Reliable differences (at the 0.01 probability level), as determined by $t$ tests on the means and standard errors given in Table 1, are as follows. (Identical conclusions follow from an analysis of the results in terms of $d^{\prime}$, a measure of sensitivity from the theory of signal detection that is free of the effects of response biases if the underlying assumptions are met. ${ }^{47,57}$ ) When the $0.75^{\prime}$ test stimulus was used, the mean of frequencyof-seeing curve (i.e., the threshold) for AOS1 was higher than those of the control subjects, and the slope and asymptote are lower. However, only the small spot produced reliable differences between AOS1 and the control subjects in the slopes and asymptotes of the frequency-of-seeing curves, and this difference between the results with these two different spots is statistically reliable. Because the false-positive rates of AOS1 did not differ reliably from those of the control subjects, these thresholds are valid estimates of sensitivity.

The larger test spot produced a threshold difference between AOS1 and the control subjects that was $40 \%$ greater $(P<0.01)$ than for the smaller spot. Hence, AOS1 shows less spatial summation than the control subjects, possibly because of lack of a complete cone mosaic, but we cannot rule out other abnormalities of his visual system that are independent of this loss of cones.

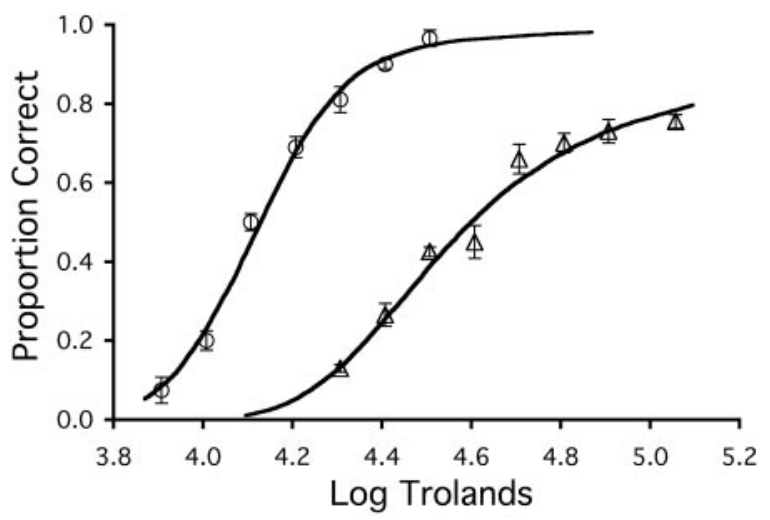

FIGURE 5. The frequency-of-seeing data for AOS1 (open triangles) and AOS2 (open circles) with the corresponding frequency-of-seeing curves generated by the model described in the text.
The data from the other color-deficient control subjects (AOS7 and AOS8) do not differ reliably from those of the trichromatic subjects in any way. When AOS7 and AOS8 are compared with AOS1, only the asymptotes for the $0.75^{\prime}$ data and the means for the $7.5^{\prime}$ data differ reliably $(t=17.3$ and 29.2 , respectively; $P<0.05$ in both cases); the decrease in the number of reliable differences may be due to the fact that the differences between AOS1 and two subjects (the two dichromats) must be greater to be statistically reliable than differences between AOS1 and seven subjects (all the control subjects).

These results show that some of the sensitivity differences between AOS1 and the control subjects show up only when small spots are used. Next we examined whether the difference could be accounted for on the basis of the differences in retinal topography.

\section{Results of the Model}

Figure 5 shows that the differences between the frequency-ofseeing curves of AOS1 and AOS2 for the small spot could be quantitatively accounted for by a model that incorporated the specific cone topographies of the respective retinas. The only differences between the models that produced these two curves were the respective subjects' cone topographies, pointspread functions, and pattern of eye movements and the associated values of the two free parameters. The subjects' pointspread functions and eye movements could be interchanged without eliminating the difference between the curves; hence, the difference in the curves was attributed to the difference in cone topographies.

The next question was whether the particular difference between retinal topographies that accounted for the difference in results lay in the number of microscotomas. Figure 6a shows the effects of varying the number of microscotomas: this family of curves resulted from running the model on the representation of the retina of AOS1 after removing varying numbers of cones (i.e., in the top two curves, missing cones have been replaced for the purposes of these tests). Free parameters are identical for all curves. Finer spacing of the curves near $72 \%$ remaining cones showed that an increase to $78 \%$ or a decrease to $67.7 \%$ produced a curve that differed reliably from the data (0.05 level, F test). This corresponded to a maximal difference in frequency-of-seeing of $0.17 \mathrm{log}$ unit. According to the model, then, the proportion of the full complement of cones remaining in the retina of AOS1 was within this range; the value estimated anatomically was near the center of this range. ${ }^{13}$ 
a

Figure 6. Model predictions assuming different proportions of remaining cones. (a, b) Model-generated curves for different proportions of a full complement of cones. From top to bottom the proportions are: $100 \%$, $85 \%, 72 \%, 55 \%, 40 \%$, and $25 \%$. Open triangles: measured frequency-ofseeing for AOS1 (a); open circles: measured frequency-of-seeing for AOS2 (b). (c) Model estimates with (solid curves) and without (dashed curves) adaptive optics for AOS1. The left curve of each pair represents the estimates for a $100 \%$ cone complement, and the right curve represents the estimates for the $72 \%$ actually present in the retina. (d) Maximum of the frequency-of-seeing curves for the retinas of AOS1 and AOS2 versus the proportion of cones present.

$b$
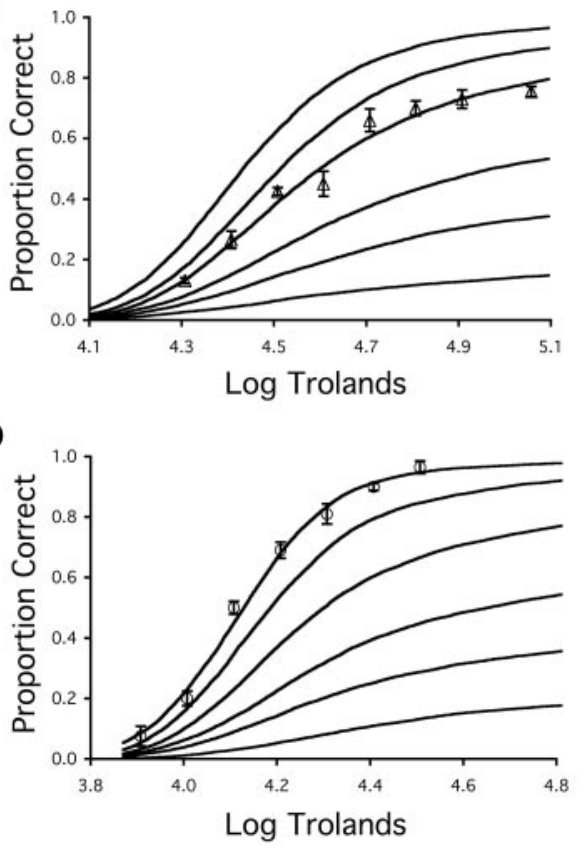

C

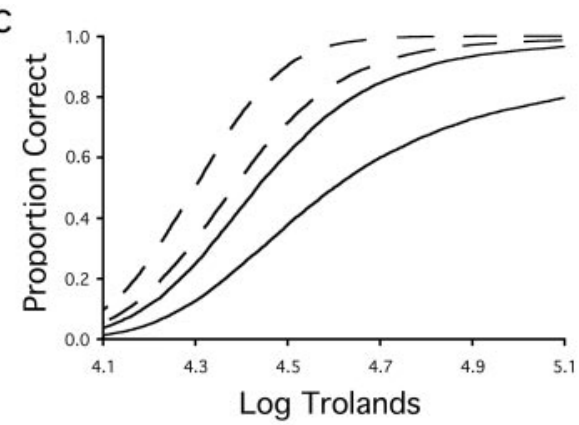

d

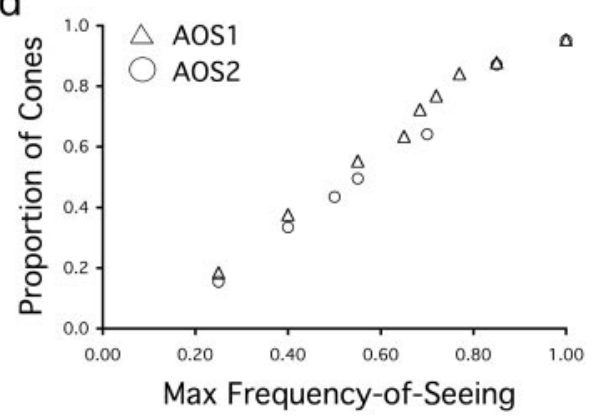

For comparison, Figure $6 \mathrm{~b}$ shows the corresponding family of curves for the retina of AOS2. These families of curves show that the differences between the frequency-of-seeing curves of AOS1 and AOS2 in Figure 4 were quantitatively attributable to the microscotomas in the retina of AOS1.

The two families of curves in Figures $6 a$ and $6 \mathrm{~b}$ differed from one another. In other words, even if AOS1 had a full complement of cones, his frequency-of-seeing curve would not be the same as that of AOS2. Such differences limit the accuracy with which one can use such curves to estimate the proportion of cones missing. To get an idea of how greatly these differences affect attempts to estimate the proportion of missing cones from the curves derived from psychophysical observations, we have plotted in Figure $6 \mathrm{~d}$ the proportion of missing cones as a function of the maximum of the frequencyof-seeing curves for the two subjects. The greatest difference between corresponding curves is $0.05 \mathrm{log}$ unit. A greater number of retinas will be needed to estimate the distribution of differences among subjects, but these preliminary data suggest that one can estimate the proportion of missing cones from the frequency-of-seeing curves to within approximately $5 \%$.

\section{Discussion}

\section{Detecting Microscotomas}

In aggregate, these results show that the anomalies that show up as gaps in the high-resolution images of the retina of AOS1 are likely to be insensitive regions as well, supporting the initial interpretation of these gaps as previous loci of cones that have become nonfunctional or possibly have died. ${ }^{13}$ Although others have used small test spots to study the contributions of the retinal mosaic to vision, ${ }^{18,33-42,58}$ it is only by correcting the eye's aberrations that one can fully exploit the advantages of using small spots. ${ }^{58}$ First, it is clear that use of large spots fails to detect microscotomas such as are present in the retina of AOS1: as mentioned, standard clinical perimetry with a $26^{\prime}$ spot revealed no deficit in the visual fields of AOS1. In addition, our $7.5^{\prime}$ spots revealed nothing that could be attributed to microscotomas, and even the smallest spots used in standard perimetry (Goldman size I, 6.6' in diameter) failed to reveal the loss of an estimated $85 \%$ to $92 \%$ of cones in patients with Stargardt disease. ${ }^{37}$ Taken together, these data point to the need to use small spots to detect scattered loss of cones.

The next question is how small they have to be. To evaluate this, we ran our model simulation using the natural pointspread function of AOS1, without correction of the higherorder aberrations, to estimate AOS1's performance without adaptive optics. Results are shown as the dashed curves in Figure $6 \mathrm{c}$, along with the simulations obtained when adaptive optics were used (the solid curves, taken from Fig. 6a). The right curve of each pair shows the results using AOS1's actual retinal mosaic, and the other was obtained by filling the lacunae with cones, representing a normal retina with a $100 \%$ complement of cones. The differences between the right and left curves in each pair are attributed to the missing cones, and it is only on the basis of these differences that the gaps left by the missing cones can be detected psychophysically.

To quantify these results and to compare them with the data from the control subjects, we fitted a cumulative Gaussian curve to the curves in Figure 6c (nearly a perfect fit), as was done with the psychophysical data. These results show that the main benefit of adaptive optics shows up in the asymptote of the frequency-of-seeing curve. Without adaptive optics, the difference between the asymptotes of the curves for $72 \%$ and $100 \%$ mosaics was 0.027 , less than the variability of asymptotes among the control subjects, as represented by the SD of the asymptotes in Figure 4. However, when adaptive optics were used, the difference between asymptotes was 0.17 , more than six times greater than without adaptive optics and more than four times the SD for the control subjects. However, the means and the spread parameters $(\sigma)$ of the curves were comparable regardless of whether adaptive optics were used. Thus, although it is possible that without adaptive optics the loss of $30 \%$ of the cones could have been detected on the basis of the means and spread parameters, adaptive optics does offer an enormous increase in sensitivity and precision (discussed in "Adaptive-Optics Microperimetry: Potential as a Research Tool") through its effect on the height of the frequency-ofseeing curves. 
Moreover, the hope of detecting such losses without adaptive optics is damped by the results of Geller and Sieving. ${ }^{37}$ Using a test spot that subtended $1.125^{\prime}$ before blurring by the eye, they observed decreased sensitivity and a decreased slope of the frequency-of-seeing curves with these patients, as we observed with AOS1, but no measurable effect on the asymptote. However, quantitatively, the effects on the slopes were much smaller than those reported. The slopes of curves from two of their patients, who were estimated to have lost $85 \%$ to $88 \%$ of their cones, corresponded to a $15 \%$ loss in our study (Figure 6). In the other two patients, an estimated loss of $85 \%$ to $88 \%$ of the cones yielded slopes corresponding to $28 \%$, and $55 \%$ losses in our study (Figure 6). One cannot know whether the slope of the frequency-of-seeing curve of AOS1 would have been abnormal in their experiment, but if the curves of two patients who have lost $85 \%$ of their cones are barely abnormal (i.e., corresponding to a $15 \%$ loss), then the abnormality of someone with only a $30 \%$ loss is unlikely to be detected. The smaller effects observed by Geller and Sieving ${ }^{37}$ might have resulted from optical aberrations they were unable to correct.

\section{Adaptive-Optics Microperimetry: Potential as a Research Tool}

The technique outlined here serves not only to detect the loss of cone function but holds promise as a means of measuring the amount of loss. Given that the shape of the frequency-ofseeing curve depends on the proportion of cones lost (Fig. 6), it can be used to estimate the magnitude of functional loss, perhaps with a precision within 5\%. This might be valuable in trying to monitor progressive loss of cone function in patients with cone degeneration, especially in those in whom functional loss might precede visible signs of loss in retinal images. Note that by testing with a wavelength to which both $\mathrm{M}$ - and L-cones are equally sensitive, there is no difference between them as far as this test is concerned. Any cone loss, whether specific to a particular cone class or randomly distributed, should reveal itself to these test stimuli.

This technique may also hold promise as a means of detecting small scotomas caused by other anomalies, such as the loss of ganglion cells that occurs in glaucoma. We speculate here that most techniques for detecting missing cones are hampered by redundancy in the representation of the stimuli in the receptor layer. The detection of missing ganglion cells is hindered not only by the same source of redundancy but also by redundancy associated with overlapping receptive fields. If one ganglion cell is lost, others continue to respond. The total signal transmitted may be smaller, and the loss of a particular ganglion cell may change the properties of the signal transmitted, but the loss of a ganglion cell fails to produce a complete scotoma, as the loss of a single cone does. Nevertheless, the stimuli used here reduce the redundancy that limits the effectiveness of such tests and are likely to increase sensitivity to the loss of ganglion cell activity.

\section{Acknowledgments}

The authors thank Peter Bex (University College, London), Li Chen (University of Rochester), Mina Chung (University of Rochester), Syndee Givre (University of North Carolina), Jay Neitz (Medical College of Wisconsin), Maureen Neitz (Medical College of Wisconsin), and Jason Porter (University of Houston) for their assistance in this work.

\section{References}

1. Kerrigan-Baumrind LA, Quigley HA, Pease ME, Kerrigan DF, Mitchell RS. Number of ganglion cells in glaucoma eyes compared with threshold visual field tests in the same persons. Invest Ophthalmol Vis Sci. 2000;41:741-748.
2. Johnson CA. Early losses of visual function in glaucoma. Optom Vis Sci. 1995;72:359-370.

3. Johnson CA. Selective vs. nonselective losses in glaucoma. J Glaucoma. 1994;3:S32-S44.

4. Glovinsky Y, Quigley HA, Pease ME. Foveal ganglion-cell loss is size dependent in experimental glaucoma. Invest Ophthalmol Vis Sci. 1993;34:395-400.

5. Drum BA, Severns M, O'Leary DK, et al. Selective loss of patterndiscrimination in early glaucoma. Appl Opt. 1989;28:1135-1144.

6. Drum BA, Severns M, O'Leary DK, et al. Pattern discrimination and light detection test different types of glaucomatous damage. In: Heijl A, ed. Perimetry Update 1988/1989. Amsterdam: Kugler \& Ghedini, 1989; v. 28.

7. Sample PA. Short-wavelength automated perimetry: its role in the clinic and for understanding ganglion cell function. Prog Retin Eye Res. 2000;19:369-383.

8. Johnson CA, Samuels SJ. Screening for glaucomatous visual field loss with frequency-doubling perimetry. Invest Opbthalmol Vis Sci. 1997;28:413-425.

9. Medeiros FA, Sample PA, Weinreb RN. Frequency doubling technology perimetry abnormalities as predictors of glaucomatous visual field loss. Am J Opbthalmol. 2004;137:863-871.

10. Frisen L. High-pass resolution perimetry: central field retino-retinal correlates. Vision Res. 1995;35:293-301.

11. Curcio CA, Allen KA. Topography of ganglion-cells in human retina. J Comp Neurol. 1990;300:5-25.

12. Neitz M, Carroll J, Renner A, Knau H, Werner JS, Neitz J. Variety of genotypes in males diagnosed as dichromatic on a conventional clinical anomaloscope. Vis Neurosci. 2004;21:205-216.

13. Carroll J, Neitz M, Hofer H, Neitz J, Williams DR. Functional photoreceptor loss revealed with adaptive optics: an alternate cause of color blindness. Proc Natl Acad Sci USA. 2004;101:84618466.

14. Crognale MA, Fry M, Highsmith J, et al. Characterization of a novel form of X-linked incomplete achromatopsia. Vis Neurosci. 2004; 21:197-203.

15. Packer O, Diller LC, Verweij J, et al. Characterization and use of a digital light projector for vision research. Vision Res. 2001;41: 427-439.

16. Harrington DO, Flocks M. Visual field examination by a new tachystoscopic multiple-pattern method. Am J Opbthalmol. 1954; 37:719-723.

17. Henson DB. Visual Fields. Oxford: Oxford University Press; 1993.

18. Frisen L. New, sensitive window on abnormal spatial vision: rarebit probing. Vision Res. 2002;42:1931-1939.

19. Trick LM, Pylyshyn ZW. Why are small and large numbers enumerated differently-a limited-capacity preattentive stage in vision. Psychol Rev. 1994;101:80-102.

20. Basak C, Verhaeghen P. Subitizing speed, subitizing range, counting speed, the Stroop effect, and aging: capacity differences and speed equivalence. Psychol Aging. 2003;18:240-249.

21. Jevons WS. The power of numerical discrimination. Nature. 1871; 3:281-282.

22. Kaufman EL, Lord MW, Reese TW, Volkmann J. The discrimination of visual number. Am J Psychol. 1949;62:498-525.

23. Sharma V, Levi DM, Klein SA. Undercounting features and missing features: evidence for a high-level deficit in strabismic amblyopia. Nat Neurosci. 2000;3:496-501.

24. Putnam NM, Hofer HJ, Doble N, Chen L, Carroll J, Williams DR. The locus of fixation and the foveal cone mosaic. J Vis. 2005;5: 632-639.

25. Artal P, Chen L, Fernández EJ, Singer B, Manzanera S, Williams DR. Neural compensation for the eye's optical aberrations. J Vis. 2004; 4:281-287.

26. Hofer H, Chen L, Yoon GY, Singer B, Yamauchi Y, Williams DR. Improvement in retinal image quality with dynamic correction of the eye's aberrations. Opt Express. 2001;8:631-643.

27. Roorda A, Williams DR. The arrangement of the three cone classes in the human eye. Nature (Lond). 1999;397:520-522.

28. Hofer H, Carroll J, Neitz J, Neitz M, Williams DR. Organization of the human trichromatic cone mosaic. J Neurosci. 2005;25:96699679. 
29. MacLeod DIA, Williams DR, Makous W. A visual nonlinearity fed by single cones. Vision Res. 1992;32:347-363.

30. Chen B, Makous W, Williams DR. Serial spatial filters in vision. Vision Res. 1993;33:413-427.

31. He S, Macleod DIA. Contrast-modulation flicker: dynamics and spatial resolution of the light adaptation process. Vision Res. 1998;38:985-1000.

32. Qi X. Spatial Summation and Antagonism of Foveal Cone Signals at Different Illuminances on the Human Retina. Rochester, NY: University of Rochester; 1997. Dissertation.

33. Cicerone CM, Nerger JL. The density of cones in the fovea centralis of the human dichromat. Vision Res. 1989;29:1587-1595.

34. Cicerone CM, Nerger JL. The relative numbers of long-wavelengthsensitive to middle-wavelength-sensitive cones in the human fovea centralis. Vision Res. 1989;29:115-128.

35. Nerger JL, Cicerone CM. The ratio of L-cones to M-cones in the human parafoveal retina. Vision Res. 1992;32:879-888.

36. Otake S, Cicerone CM. L and $\mathrm{M}$ cone relative numerosity and red-green opponency from fovea to midperiphery in the human retina. J Opt Soc Am A Opt Image Sci Vis. 2000;17:615-627.

37. Geller AM, Sieving PA. Assessment of foveal cone photoreceptors in Stargardt's macular dystrophy using a small dot detection task. Vision Res. 1993;33:1509-1524.

38. Vimal RLP, Pokorny J, Smith VC, Shevell SK. Foveal cone thresholds. Vision Res. 1989;29:61-78.

39. Wesner MF, Pokorny J, Shevell SK, Smith VC. Foveal cone detection statistics in color-normals and dichromats. Vision Res. 1991; 31:1021-1037.

40. Williams DR, MacLeod DIA, Hayhoe MM. Punctate sensitivity of the blue sensitive mechanism. Vision Res. 1981;21:1357-1376.

41. Marriott FHC. The foveal absolute threshold for short flashes and small fields. J Physiol (Lond). 1963;169:416-423.

42. Krauskopf J, Srebro R. Spectral sensitivity of color mechanisms: derivation from fluctuations of color appearance near threshold. Science. 1965;150:1477-1479.

43. Watson AB. Visual detection of spatial contrast patterns: evaluation of five simple models. Opt Express. 2000;6:12-33.
44. Watson AB, Ahumada AJ Jr. A standard model for foveal detection of spatial contrast. J Vis. 2005;5:717-740.

45. Bex PJ, Makous W. Spatial frequency, phase and the contrast of natural images. J Opt Soc Am A Opt Image Sci Vis. 2002;19:10961106.

46. Quick RF. A vector-magnitude model of contrast detection. Kybernetik. 1974;16:65-67.

47. Van Trees HL. Detection, Estimation, and Modulation Theory, Part I: Detection, Estimation, and Linear Modulation Theory. New York: Wiley; 1968.

48. Green DM, Swets JA. Signal Detection Theory and Psychophysics. Huntington, NY: Robert E. Krieger Publishing Company; 1974.

49. MacMillan NA. Signal detection theory. In: Wixted J, ed. Stevens' Handbook of Experimental Psychology. 3rd ed. New York: John Wiley \& Sons, Inc., 2002.

50. Curcio CA, Sloan KR. Packing geometry of human cone photoreceptors: variation with eccentricity and evidence for local anisotropy. Vis Neurosci. 1992;9:169-180.

51. Curcio CA, Sloan KR, Kalina RE, Hendrickson AE. Human photoreceptor topography. J Comp Neurol. 1990;292:497-523.

52. Snodderly DM, Weinhaus RS, Choi JC. Neural-vascular relationships in central retina of macaque monkeys (Macaca fascicularis). J Neurosci. 1992;12:1169-1193.

53. Conrath J, Giorgi R, Raccah D, Ridings B. Foveal avascular zone in diabetic retinopathy: quantitative vs qualitative assessment. Eye. 2005;19:322-326.

54. Hendley $\mathrm{CD}$. The relation between visual acuity and brightness discrimination. J Gen Physiol. 1948;31:433- 457.

55. Graham $\mathrm{CH}$, Bartlett NR. The relation of the size of stimulus and intensity in the human eye, III: the influence of area on foveal intensity discrimination. J Exp Psychol. 1940;27:149-159.

56. Willmer EN. The relationship between area and threshold in the central fovea for lights of different colours. Q J Exp Psychol. 1950;2:53-59.

57. Green DM, Swets JA. Signal Detection Theory and Psychophysics. New York: Wiley; 1966.

58. Hofer H, Singer B, Williams DR. Different sensations from cones with the same photopigment. J Vis. 2005;5:444-454. 